\title{
Analyse des écoulements instationnaires par interférométrie holographique numérique couleur (de la plaque holographique au numérique)
}

\author{
Jean-Michel DESSE \\ ONERA, The French Aerospace Lab \\ Jean-Michel.Desse@onera.fr \\ $>$ Pascal PICART \\ LAUM UMR CNRS 661 - ENSIM \\ Pascal.Picart@univ-lemans.fi
}

I I est bien connu que l'interférométrie holographique monochromatique ou polychromatique adaptée à l'analyse des écoulements présente une très haute résolution spatiale lorsque des plaques holographiques sont utilisées en réflexion ou en transmission. Les inconvénients de cette méthode résident dans le traitement chimique des plaques, le contrôle de l'épaisseur de la gélatine et le repositionnement des hologrammes dans le montage optique. L'interférométrie holographique numérique couleur décrite ici permet d'obtenir des résultats tout à fait comparables à ceux obtenus en holographique analogique. La technique est basée sur l'enregistrement de microfranges d'interférences d'un état de référence, soufflerie arrêtée, et d'un état de déformation, soufflerie en fonctionnement. Un traitement par transformée de Fourier 2D directe et inverse permet de filtrer l'information pour reconstruire les cartes de phase et d'amplitude. Les cartes de différence de phase et d'intensité lumineuse sont calculées pour être comparées aux interférogrammes analogiques obtenus par la méthode d'interférométrie holographique utilisant des plaques holographiques panchromatiques par réflexion. Le cas test choisi concerne l'écoulement instationnaire autour d'un cylindre en écoulement subsonique dans lequel l'évolution du champ de la masse volu- mique est déterminée pour un cycle de I'allée alternée et pour un nombre de Mach de 0,45.

\section{Pourquoi développer l'holographie numérique pour l'étude des écoulements?}

Dans le domaine de la mécanique des fluides, les codes CFD ont besoin d'être initialisés et validés par des données expérimentales de plus en plus précises dans le temps et/ou l'espace. C'est pourquoi l'ONERA et le LAUM ont développé des outils métrologiques non intrusifs permettant d'accéder à des mesures globales (mesures de champs complets) ayant des résolutions spatiales et temporelles élevées. Dans le cas d'étude d'écoulements aérodynamiques complexes, les techniques développées doivent permettre d'accéder à des données instationnaires (temps réel) et, comme les objets à analyser sont des objets «transparents ", la grandeur à mesurer est l'indice de réfraction du milieu. La relation de Gladstone Dale permet ensuite d'accéder à la masse volumique.

Il y a quelques années, I'ONERA a développé pour l'analyse des écoulements instationnaires une métrologie optique basée sur l'interférométrie holographique analogique à trois longueurs d'onde utilisant des supports holographiques panchromatiques par transmission et par réflexion [1, 2]. Le principal inconvénient de cette dernière métrologie réside dans le contrôle de l'épaisseur de la gélatine des hologrammes pendant le développement des plaques et l'efficacité de diffraction de l'hologramme qui doit être voisine de $50 \%$ simultanément pour les trois longueurs d'onde utilisées. Pour éviter ces problèmes et s'affranchir des supports holographiques, l'interférométrie holographique numérique à trois longueurs d'onde a été développée au département de l'aérodynamique appliquée de l'ONERA-Lille, en collaboration avec I'université du Maine au Mans. Une étude de faisabilité a été réalisée sur une flamme de bougie et les principaux résultats peuvent être trouvés dans la référence [3]. La technique a ensuite été appliquée à un cas concret d'écoulement instationnaire pour être comparée aux résultats trouvés en holographie à plaque.

Avant: la plaque holographique enregistrait les réseaux de diffraction

\section{Un montage optique apparenté au montage classique Lippman-Denisyuk}

Pour analyser l'écoulement instationnaire qui se développe en aval d'un cylindre placé transversalement dans un écoulement subsonique, I'ONERA a tout d'abord développé l'interférométrie holographique en temps réel et en vraies couleurs de type "Denisyuk" dont le montage optique est présenté sur la figure 1. Un cube à séparation de polarisation est inséré entre le filtre spatial et I'hologramme. La source lumineuse est constituée par trois raies laser, une rouge, une verte et une bleue, fournies par un laser argon et krypton pour la raie rouge du krypton à $647 \mathrm{~nm}$ et par deux lasers DPSS (laser solide pompé par diode) pour les raies verte et bleue $(532 \mathrm{~nm}$ et $457 \mathrm{~nm})$. Le filtre spatial, constitué d'un objectif de microscope (x 60), focalise la lumière dans un trou de $25 \mu \mathrm{m}$ pour que I'hologramme soit éclairé par un faisceau de lumière divergente de diamètre $80 \mathrm{~mm}$ environ. Le miroir plan $\gg$ 


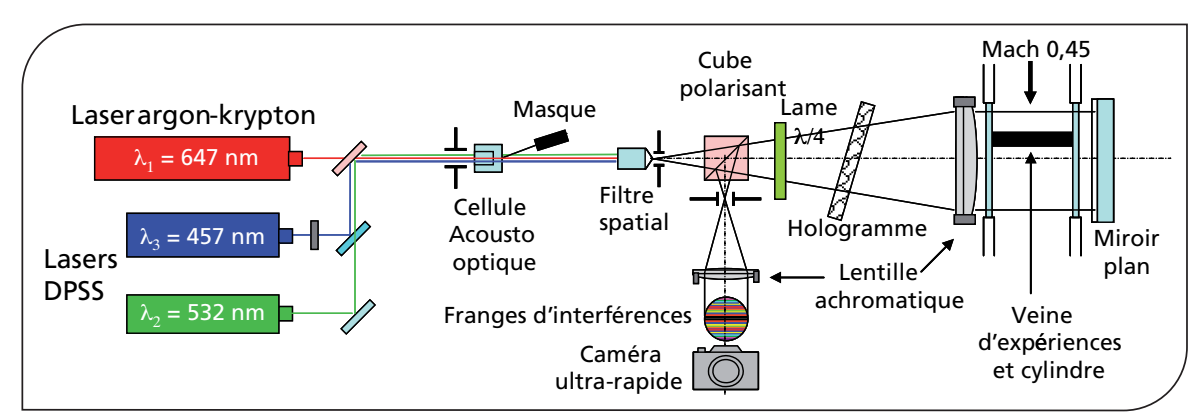

Figure 1. Interféromètre holographique en vraies couleurs et en temps réel de type « Denisyuk».

positionné juste derrière la veine d'expériences renvoie les faisceaux lumineux vers I'hologramme. L'hologramme est alors éclairé simultanément des deux côtés de la plaque par les trois faisceaux incidents divergents et par les trois faisceaux réfléchis convergents. La lame $\lambda / 4$ associée au cube polarisant permet de faire tourner la polarisation des faisceaux laser et de renvoyer, au retour, les faisceaux vers la caméra ultrarapide pour I'enregistrement du phénomène.

Ceci étant, I'hologramme est éclairé une première fois en absence d'écoulement dans la veine d'expériences, développé et replacé exactement à la position qu'il occupait lors du premier enregistrement. L'analyse de l'efficacité de diffraction des trois réseaux d'interférences enregistrés dans la gélatine montre que les valeurs sont voisines de $50 \%$ pour les trois longueurs d'onde utilisées. II en ressort que, lorsque la plaque holographique sera repositionnée dans le montage optique, $50 \%$ de la lumière incidente sera réfléchie par l'hologramme et constituera les trois ondes de référence au niveau de la caméra et les autres $50 \%$ traverseront I'hologramme, puis la veine d'expériences et enfin seront renvoyés par le miroir plan et constitueront les ondes de mesure. Le montage présente donc une sensibilité doublée car la veine d'expériences est traversée deux fois. Si la veine d'expériences est au repos, on observera trois teintes plates sur l'écran, une teinte rouge, une verte et une bleue, donc une teinte blanche. Si un écoulement existe dans la veine d'expériences, des franges $d$ 'interférences en couleur visualisant les lement apparaîtront sur l'écran. Ce montage optique présente des avantages et des inconvénients. Tout d'abord, les longueurs de cohérence des trois raies laser doivent être au moins égales à deux fois la distance entre la plaque holographique et le miroir plan, ici typiquement de l'ordre du mètre. II n'y a donc pas de problème avec les raies bleue et verte du DPSS, puisque cette distance est de l'ordre de $5 \mathrm{~m}$ et $40 \mathrm{~m}$ respectivement. Concernant la raie rouge du krypton, la longueur de cohérence de cette raie a été augmentée en insérant un étalon Fabry Pérot dans la cavité laser. Le principal inconvénient réside dans le fait qu'il n'est pas possible d'équilibrer l'intensité lumineuse des faisceaux de référence et de mesure. Enfin, le support de plaque variations d'indice de réfaction de l'écou- holographique est légèrement incliné pour que I'hologramme soit facilement repositionné. II faut noter que les deux hublots de la soufflerie ont été traités anti-reflet pour éviter les interférences parasites provoquées par l'épaisseur de verre de ces hublots. La figure 2 montre deux photographies du montage dans la soufflerie transsonique de Lille.

\section{Les résultats obtenus sur le cylindre}

La maquette testée est un cylindre circulaire de $20 \mathrm{~mm}$ de diamètre placé transversalement dans la veine d'expériences de la soufflerie transsonique de Lille. Le nombre de Mach infini amont a été fixé à 0,45 . L'interférogramme de référence est réalisé avec un temps de pose de 0,15 seconde.

L'énergie lumineuse au niveau de la plaque holographique est de l'ordre de $400 \mu \mathrm{J} / \mathrm{cm}^{2}$ pour chaque raie. Les interférogrammes sont enregistrés par une caméra ultrarapide constituée d'un tambour tournant et d'un petit miroir central à huit facettes tournant en sens inverse du tambour. La cadence d'enregistrement est de 35000 images par seconde et le temps d'exposition de chaque interférogramme est de 750 nanosecondes. La figure 3 montre une succession d'interférogrammes enregistrés en teinte de fond uniforme avec un $\Delta \mathrm{T}$ de $117 \mu \mathrm{s}$. On voit que les tourbillons sont représentés par des lignes concentriques de couleurs différentes. Chaque couleur représente en fait une ligne isochore de masse volumique. Le problème est maintenant de savoir s'il est possible d'obtenir les mêmes résultats en développant une technique d’holographie numérique.
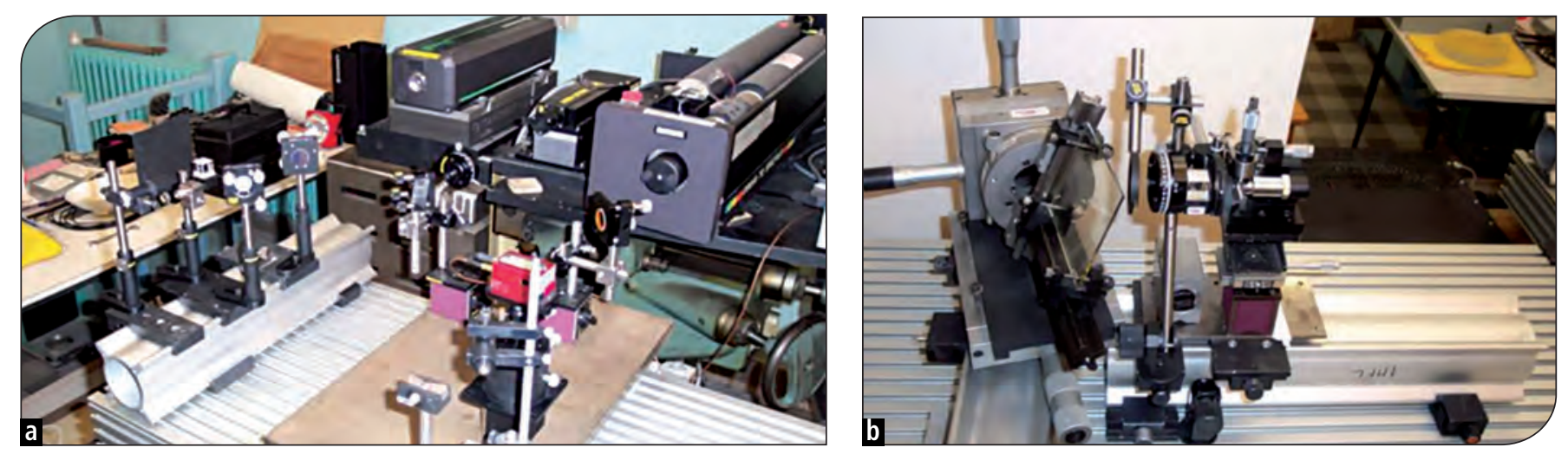

Figure 2. Photographie du montage optique en soufflerie : (a) collimation des sources lasers ; (b) support de la plaque holographique. 


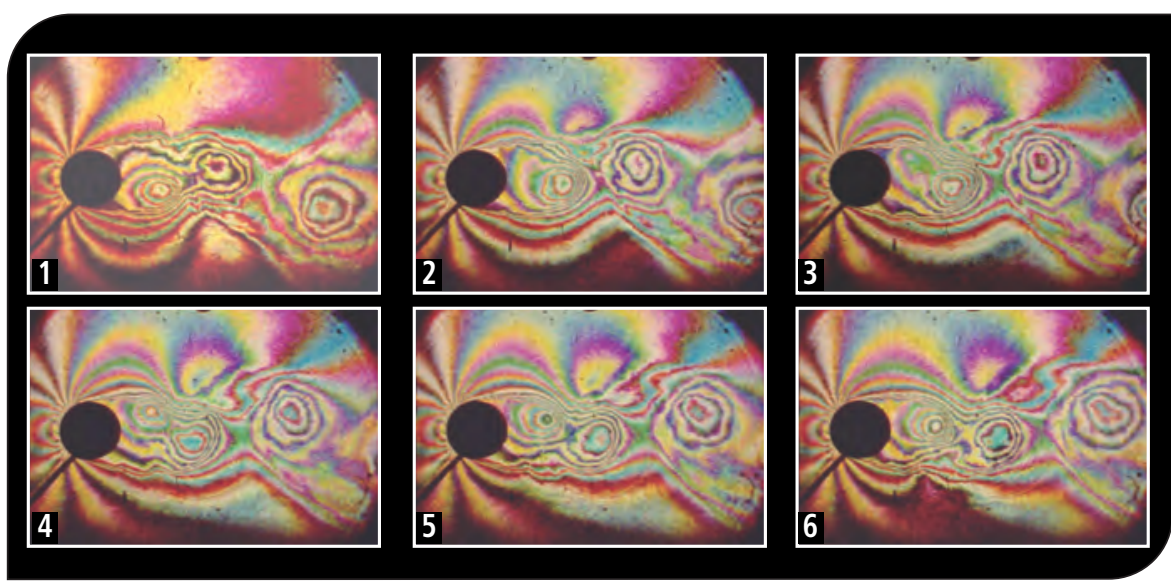

Figure 3. Interférogrammes successifs de l'écoulement autour du cylindre - Mach 0,45.

\section{Maintenant : le capteur 3CCD enregistre directement les interférences}

Il existe deux possibilités pour analyser les objets transparents en mécanique des fluides par une méthode d'interférométrie holographique numérique couleur. La première consiste, tout comme en mécanique des solides, à former l'image des ondes de mesure traversant la veine d'expériences sur un verre dépoli. La lumière diffractée par le verre dépoli interfère sur le capteur d'enregistrement avec trois ondes de référence. Dans ce cas, le capteur enregistre une image de speckle en couleur et la phase des trois hologrammes, rouge, vert et bleu, est calculée par transformation de Fresnel dans l'ordinateur. Le problème est que la taille et la localisation des images dans le plan de Fresnel dépend de la longueur d'onde. II n'est donc pas simple de les superposer pour obtenir une information couleur.

La seconde consiste à former directement sur le capteur des microfranges d'interférences produites par les trois ondes de mesure qui ont traversé la veine d'expériences et les trois ondes de référence. Ces microfranges d'interférences seront utilisées comme fréquences porteuses spatiales de l'information. C'est cette solution qui a été choisie.

\section{Le tri CDD, un capteur idéal pour l'holographie à trois longueurs d'onde}

Le choix du capteur d'enregistrement (dimension du pixel, nombre de pixels, présence d'un filtre de Bayer ou pas) est

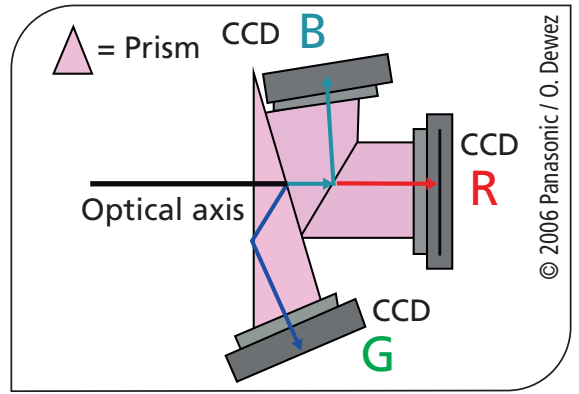

Figure 4. Caractéristiques du capteur tri-CDD.

très important puisqu'il conditionne la résolution des cartes de différence de phase obtenues. Après avoir comparé les possibilités des capteurs type Foveon, vidéo mono trame, tramé, CMOS et CCD équipé d'un filtre de Bayer et $3 C C D$, c'est le capteur 3CCD de chez Hamamatsu qui a donné les meilleurs résultats. Comme on peut le voir sur la figure 4, chaque longueur d'onde est directement renvoyée sur un CCD dont la courbe de transmission est pratiquement centrée sur la couleur du laser. Ceci est très important car l'analyse spectrale 2D faite sur chacune des voies montre un spot unique correspondant à la fréquence des microfranges d'interférence à la longueur d'onde de l'enregistrement. II n'y a aucune information parasite ce qui facilite grandement l'opération de filtrage.

\section{Un montage optique facile à régler}

Le schéma optique relatif à I'holographie numérique (figure 5) comporte $\gg$

\section{\begin{tabular}{l} 
- LA RÉPONSE À vOS APPLICATIONS : SPECTROSCOPIE, HOLOGRAPHIE, LIDAR DOPLER \\
$\begin{array}{ll}\text { - } 700 \mathrm{~mJ} \text { à } 1064 \mathrm{~nm} & \text { Principales caractéristiques } \\
\text { - Largeur de raie : } 0,005 \mathrm{~cm}^{-1} & \text { - Générateurs d'harmoniques interchangeables } \\
\text { - Profil temporel lisse } & \text { - Pas d'installation } \\
\text { - Injection par laser à fibre CW à haute stabilité } & \text { - Refroidissement air/eau autonome }\end{array}$ \\
\hline Pour plus d'informations, contactez-nous au 0169291700 ou par e-mail à quantel@quantel.fr - www.quantel-laser.com \\
\hline
\end{tabular}}




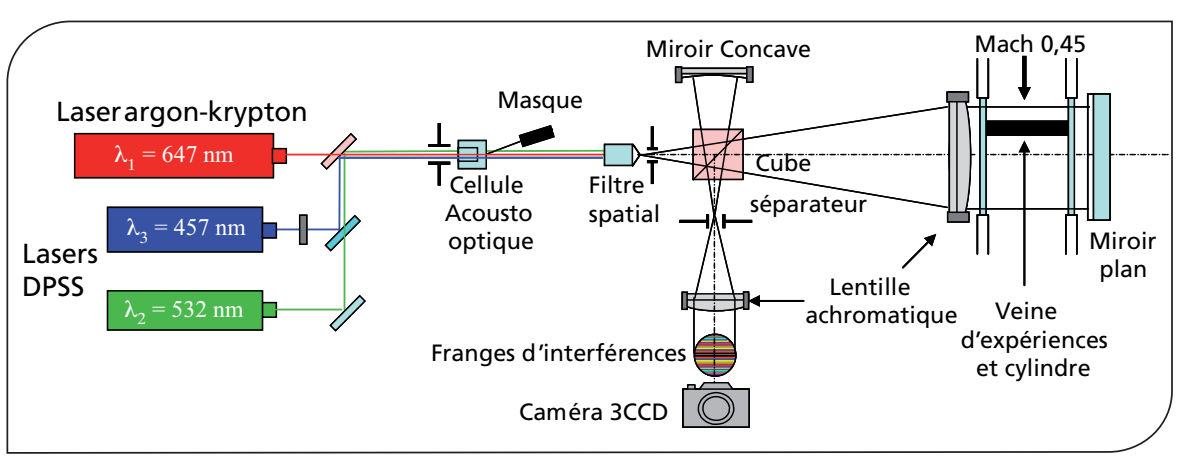

Figure 5. Interférométrie holographique numérique à trois longueurs d'onde.

un cube séparateur qui divise les trois faisceaux incidents pour former les trois ondes de référence renvoyées par le miroir concave sur la caméra et les trois ondes de mesure qui traversent la veine d'expériences. Le filtre spatial positionné à la distance focale de la grande lentille achromatique permet d'éclairer la veine d'expériences en trois faisceaux de lumière parallèle. Le miroir plan placé juste derrière la veine renvoie les faisceaux sur le cube séparateur qui les retourne vers la caméra. La seconde lentille achromatique forme l'image sur le tri-CCD et sa distance focale fixe la dimension du champ enregistré.

Lorsque les points de focalisation des ondes de référence et des ondes de mesure sont superposés dans le diaphragme qui est placé devant la caméra, on peut observer trois teintes de fond (rouge, vert et bleu) sur le CCD. Si on déplace le point de focalisation des faisceaux de référence dans le plan du diaphragme, on introduit des franges d'interférences rectilignes dans le champ d'observation. Ceci est obtenu très simplement en tournant le miroir concave. À ce stade, on enregistre sur le CCD les interférences lumineuses qui permettront de calculer les trois hologrammes de référence et notamment les cartes de phase de référence. Ensuite, la soufflerie est mise en route et les trois ondes de mesure sont déformées par le phénomène aérodynamique, ce qui provoque une déformation des microfranges d'interférences. Cellesci sont enregistrées de nouveau par le capteur CCD pour calculer les cartes de phase liées à la mesure. La figure 6 montre deux images des microfranges d'interférences enregistrées avec et sans
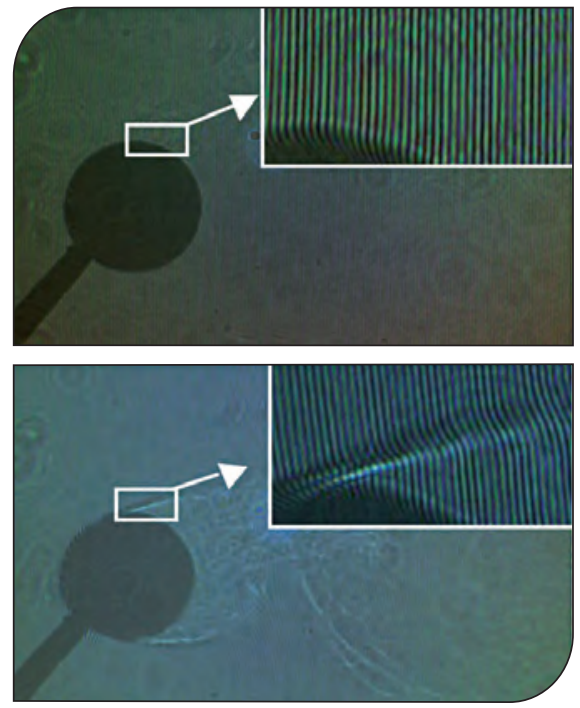

Figure 6. Enregistrement des microfranges avec et sans écoulement - Mach 0,45.

écoulement. Dans la partie zoomée, on peut voir comment se déforment les microfranges d'interférences à l'extrados du cylindre.

\section{Des résultats très concluants}

La figure 7 montre les spectres bidimensionnels de Fourier pour l'image de référence et pour l'image de mesure pour la

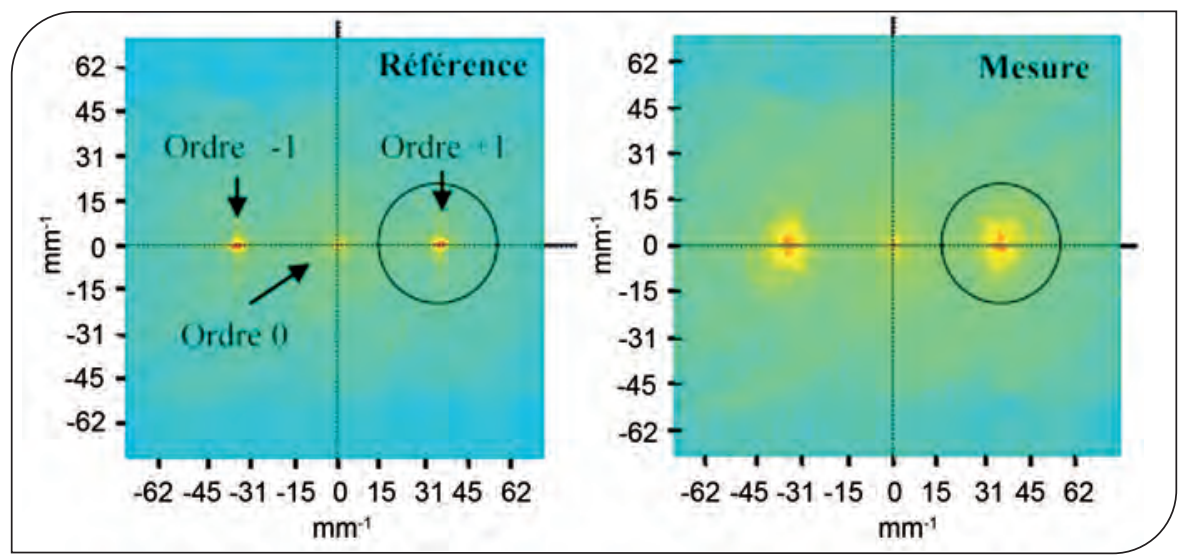

Figure 7. Localisation fréquentielle du signal et filtrage - voie bleue. verte et bleue. La figure 8 montre les cartes de différence de phase modulo $2 \pi$ et déroulée obtenues pour la voie bleue (différence entre la phase de mesure et la phase de référence). On peut distinguer qu'à l'endroit de l'émission des lignes tourbillonnaires issues de l'intrados et de l'extrados du cylindre, les sauts de phase ne sont pas très bien résolus localement. Cela est dû soit au temps de pose du cliché de $1 \mu$ s, qui est un peu long devant le temps du phénomène, soit à l'orientation des microfranges d'interférences. Les cartes d'épaisseur optique et d'indice de réfraction sont calculées à partir des cartes de différences en prenant en compte la valeur de la longueur d'onde sur chaque plan.

\section{Comparaison entre l'holographie image et I'holographie numérique}

Si on veut comparer les deux techniques d'holographie, il nous faut calculer l'intensité des franges d'interférences d'un hologramme numérique pour la comparer avec l'intensité lumineuse des franges d'interférences enregistrée par la plaque voie bleue. Les spectres de Fourier obtenus sur chaque voie sont filtrés des ordres zéro et -1 car la carte de phase n'est reconstruite qu'avec l'ordre +1 . Cela est ici facile car l'information fréquentielle relative à l'ordre +1 est bien identifiée et non parasitée du fait du choix de la caméra équipée de 3 CDD différents.

Ensuite, les cartes de phase et d'amplitude sont calculées en utilisant les parties des spectres filtrés. À noter que les zones de filtrage peuvent être choisies indépendamment pour les voies rouge,

Photoniques • 56 • novembre/décembre 2011 


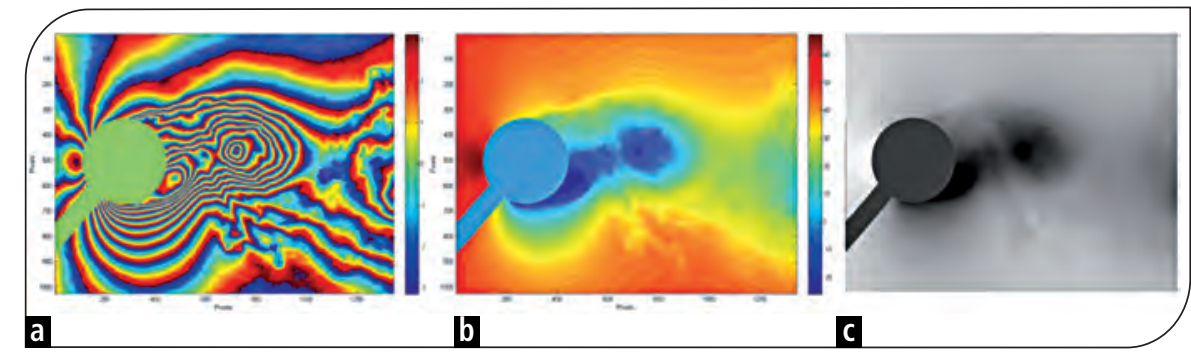

Figure 8. Traitement des cartes de différence de phase pour la voie bleue : (a) phase modulo $2 \pi$; (b) phase déroulée ; (c) carte d'épaisseur optique.

holographique. La figure 9 montre les résultats obtenus. On voit tout d'abord l'intensité lumineuse calculée plan par plan, puis la superposition des trois plans permet d'obtenir des franges en couleur et de les comparer à l'hologramme image au même instant de la position de l'allée alternée. La correspondance est assez remarquable et valide tout le processus de traitement par FFT lié à l'holographie numérique.

\section{Intérêt de la couleur en holographie}

Dans cette étude, le cylindre est équipé d'un capteur de pression instationnaire qui enregistre les fluctuations de l'allée alternée qui se développe derrière le cylindre. Il est donc possible de déclencher l'enregistrement de l'interférogramme en synchronisant ce déclenchement sur la phase de la fluctuation de pression. Pour décrire correctement un cycle complet de l'allée alternée, huit interférogrammes, décalés successivement de $45^{\circ}$, ont été enregistrés à des périodes différentes. La succession de ces huit interférogrammes permet donc de couvrir une période du phénomène. Par ailleurs, dans le cas précis de l'écoulement instationnaire autour d'un cylindre, on sait que la masse volumique au point d'arrêt (nez du cylindre) est constante et égale à la masse volumique génératrice. Il en ressort que la valeur de l'indice de réfraction au nez du cylindre doit être identique sur tous les interférogrammes. Si on regarde trois interférogrammes pris à des instants aléatoires du phénomène, on voit que la couleur au nez du cylindre n'est pas toujours la même (figure 10), ce qui signifie que des vibrations extérieures au phénomène ont fait bouger le banc optique ou les réseaux des fréquences porteuses de l'interférogramme entre l'enregistrement de l'hologramme de référence et l'enregistrement de l'hologramme de mesure. L'exemple de la figure 10 montre tout l'intérêt d'utiliser une source trichromatique. Les trois couleurs trouvées au nez du cylindre sont identifiées dans l'échelle des teintes du diagramme des $\gg$

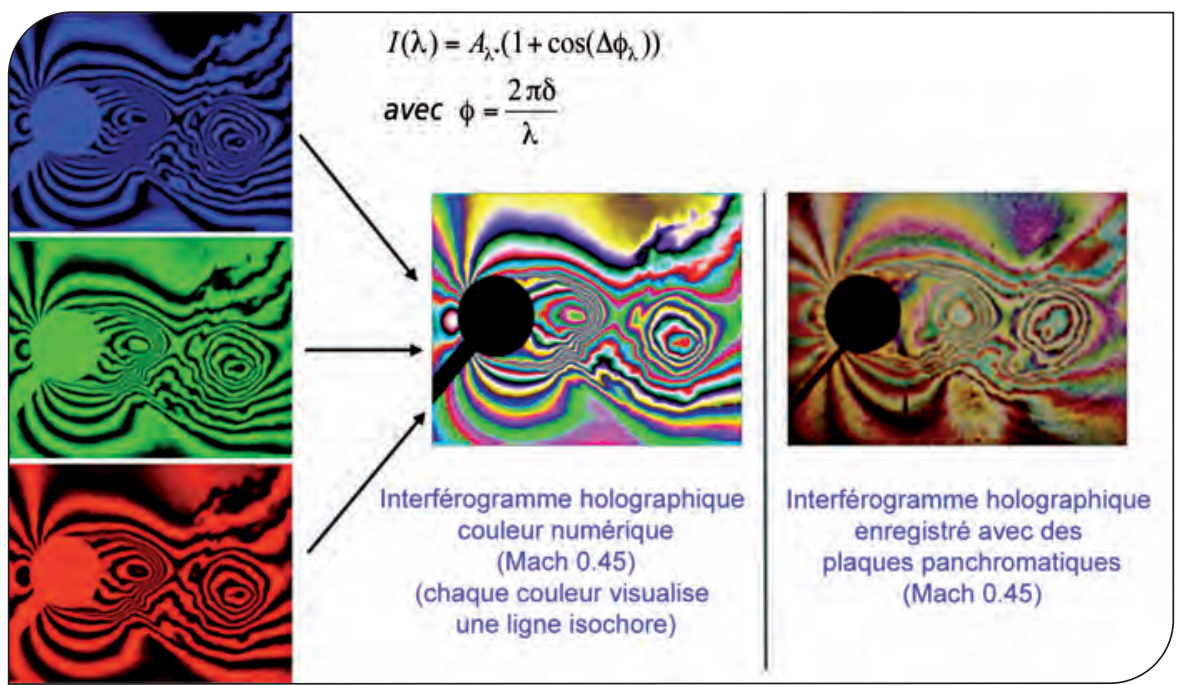

Figure 9. Comparaison entre un hologramme analogique et numérique.

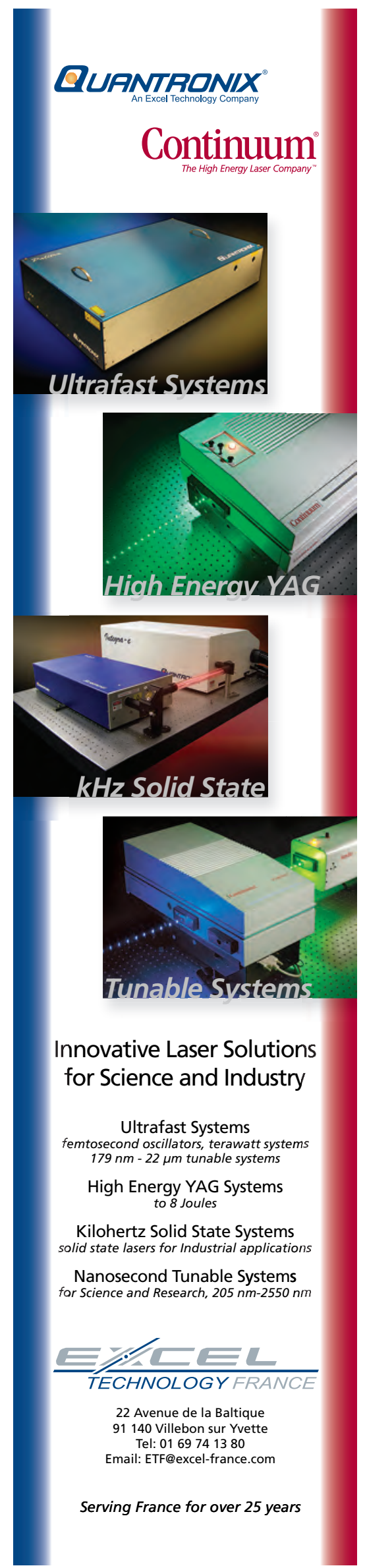

novembre/décembre 2011 • 56 P Thotoniques 


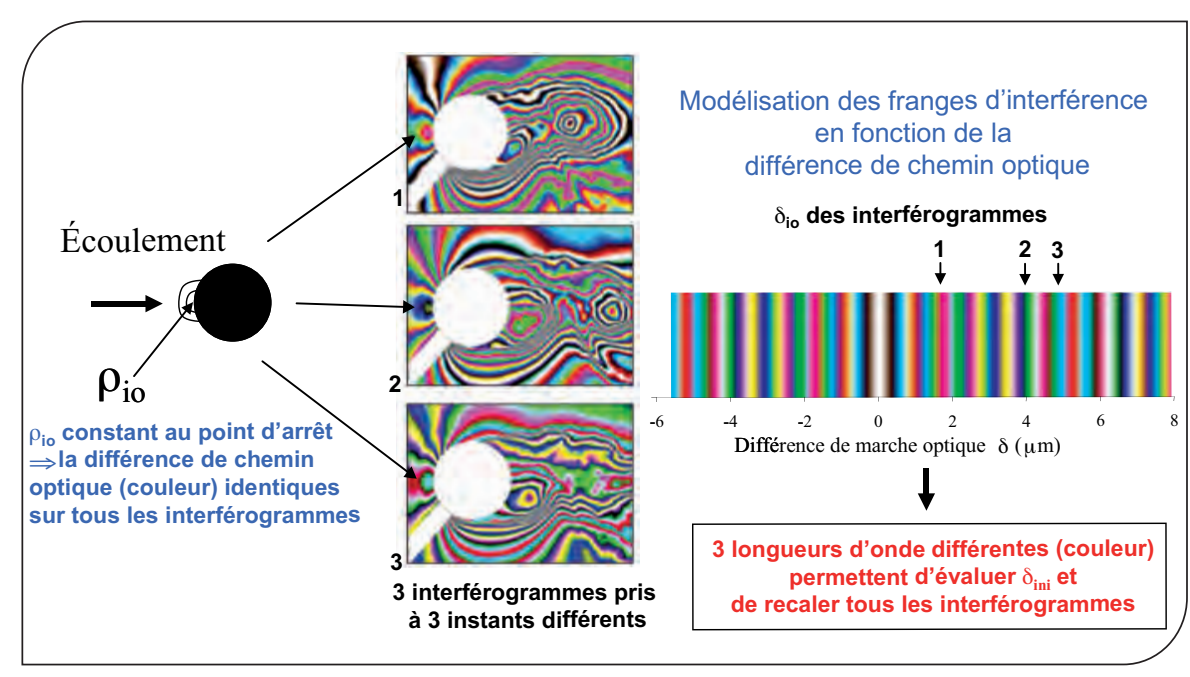

Figure 10. Recalage des interférogrammes en fonction de la couleur au point d'arrêt.

interférences lumineuses modélisées en fonction de la différence de marche. On trouve respectivement : $1,85 \mu \mathrm{m}$ pour l'interférogramme $n^{\circ} 1 ; 3,95 \mu \mathrm{m}$ pour le $n^{\circ} 2 ; 4,73 \mu$ m pour le $n^{\circ} 3$. On peut donc à partir de la connaissance de ce $\delta_{\text {ini }}$ initial recaler tous les interférogrammes entre eux.

\section{Les résultats obtenus en holographie numérique}

Un échantillonnage conditionné, basé sur l'enregistrement du signal d'un capteur de pression instationnaire implanté sur la maquette, a permis de conditionner les visualisations en fonction de la position de l'allée alternée qui se développe en aval du cylindre. Pour chaque phase analysée, une moyenne a été calculée sur dix interférogrammes pris à des périodes différentes pour obtenir le champ instantané de la masse volumique. Les cartes obtenues sur un cycle ont été également moyennées pour éva- luer le champ moyen de la masse volumique. La figure 11 montre les trois premiers interférogrammes instantanés et les cartes de champ moyenné.

\section{Perspectives et développement}

II ressort de cette comparaison entre les deux méthodes optiques que l'interférométrie holographique numérique utilisant trois longueurs d'onde comme source lumineuse et une caméra tri-CCD pourl'enregistrement des interférogrammes peut avantageusement remplacer l'interférométrie holographique numérique utilisant des plaques holographiques par transmission ou par réflexion.

Les résultats obtenus en holographie numérique ont été validés sur l'étude de l'écoulement subsonique au voisinage d'un cylindre placé transversalement dans la veine d'expériences. Le montage optique permet facilement d'introduire des fréquences porteuses spatiales qui

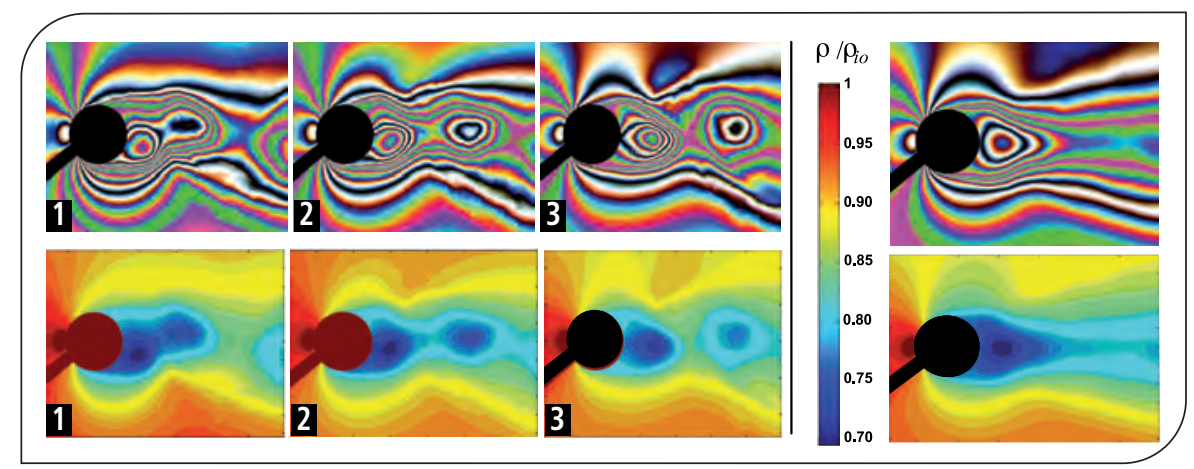

Figure 11. Évolution des franges d'interférences et de la masse volumique instantanée et moyennée $-\Delta t=76$ us. sont analysées avec et sans écoulement. Les spectres de Fourier obtenus sur chaque voie peuvent être filtrés aisément car les fréquences spatiales sont bien identifiées et non parasitées. La reconstruction des cartes de différence de phase permet d'obtenir des cartes d'indice de réfraction de très bonne qualité.

Un échantillonnage conditionné, basé sur la mesure du signal d'un capteur de pression instationnaire implanté sur la maquette, permet de discrétiser les visualisations en fonction de la position de l'allée alternée qui se développe en aval du cylindre. Pour chaque phase analysée, une moyenne a été calculée pour obtenir le champ instantané de la masse volumique. Les cartes obtenues sur un cycle ont été également moyennées pour évaluer le champ moyen de la masse volumique. De plus, l'intérêt de la couleur obtenue par une source trichromatique est clairement démontré. II offre la possibilité de recaler tous les interférogrammes entre eux car les conditions génératrices de l'écoulement sont retrouvées au point d'arrêt sur le cylindre.

Dans un avenir proche, la méthode doit être tout d'abord testée pour analyser des écoulements à très faibles masses volumiques. II s'agira de déterminer la sensibilité de la méthode à la détection de très faibles variations de phase, donc de masse volumique. Ensuite, l'écoulement autour du cylindre sera étudié pour des écoulements transsoniques et supersoniques. Cette fois, il s'agira de tester la méthode pour l'analyse des forts gradients d'indice de réfraction. Remerciements

Les auteurs remercient l'Agence nationale française pour la recherche (ANR) pour avoir financé la partie des travaux concernant l'holographie numérique sous le contrat d'Agrément n ANR 2010 BLAN 0302.

\section{Références}

[1] J. M. Desse and J.L. Tribillon. Real-time color holographic interferometry. App. Optics 41(25), 5326-5333 (2002).

[2] J.M. Desse and J.L. Tribillon. Real-time three-color reflection holographic interferometry. App. Optics 48(36), 6870-6877 (2009).

[3] J.M. Desse, P. Picart and P. Tankam. Digital threecolor holographic interferometry for flow analysis. Opt. Express 16(8), 5471-5480 (2008). 